\title{
Prospects on the Hyphenated Electrochemistry and Mass Spectrometry as a Practical Analytical Technique in the Assessment of Oxidative Drug Metabolism
}

\author{
Eslam Nouri-Nigjeh
}

Department of Pharmacology, David Geffen School of Medicine, University of California at Los Angeles, CA 900956948, USA

E-mail: enouri@mednet.ucla.edu, eslamnouri@gmail.com; Tel.: +310-825-4321

Received: September 07, 2014; Revised: September 11, 2014; Published: September 16, 2014

\begin{abstract}
For several years, electrochemistry in combination with mass spectrometry has been the subject of attention and development for studying the oxidative drug metabolism at early stages of new drug development. Though the technique could successfully imitate the in vivo oxidative drug metabolism initiated by electron transfer, it lags in the imitation of reactions initiated by either hydrogen atom transfer or oxygen atom transfer. The prospect of using electrochemistry as a practical analytical technique in the imitation of oxidative drug metabolism, therefore, relies on the extension of its utility toward covering those reactions initiated by hydrogen atom transfer and oxygen atom transfer. In this brief critical review, I discuss potential electrochemical techniques that can benefit the application of electrochemistry beyond electron transfer reactions.
\end{abstract}

\section{Keywords}

Electrochemistry; mass spectrometry; oxidative drug metabolism

\section{Introduction}

Due to the large number of drug candidates at the early stages of new drug development, there is a tangible need for a fast and facile analytical technique to assess the oxidative drug metabolism. Hyphenated electrochemistry and mass spectrometry technique has been in the center of attention for the last several years to achieve the fast and facile assessment of oxidative drug metabolism at the early stages of new drug development [1,2]. This brief review will present the current status of the technique, and possible efforts to extend its application in a more practical sense in the assessment of oxidative drug metabolism.

Cytochrome P450s are the main family of monooxygenase enzymes that are involved in phase I in vivo oxidative drug metabolism pathways [3]. The reactive pocket of these enzymes include a prosthetic iron protoporphyrin IX group that is axially anchored with a cysteine residue in the protein backbone [3]. Interaction of the substrate drug compound with a specific part of the reactive pocket triggers the activation of molecular oxygen in a chain of proton and electron transfer events to generate oxo-ferryl 
radical cation species [4]. These species are considered the main reactive species produced during the catalytic activation of molecular oxygen with Cytochrome P450s, and are highly reactive toward the oxidation of substrate drug compounds that are already located in the vicinity of the reactive pocket of the enzyme [5].

Cytochrome P450s and their main reactive species, i.e. oxo-ferryl radical cations, promote different oxidative metabolism pathways; namely, those initiated by electron transfer reaction, such as $N$ dealkylation of tertiary amine moieties, those initiated by hydrogen atom transfer, such as $O$-dealkylation pathways, and those initiated by oxygen atom transfer such as $\mathrm{N}$-oxidation pathways. There are already a great number of studies directed toward exploration of reactive species generated during catalytic activation of molecular oxygen with Cytochrome P450s, and their reactivities toward promotion of different oxidative metabolism pathways [6]. The products of oxidative drug metabolism, that are usually more hydrophilic than drug substrates, conjugate with glutathione to be secreted from the cells in second and third phases of oxidative drug metabolism [3].

Direct electrochemical oxidations, unlike reactive species generated during catalytic activation of molecular oxygen by Cytochrome P450s, can only promote oxidation reactions initiated by electron transfer reactions $[7,8]$. This means that a subset of in vivo metabolism pathways that are initiated by either hydrogen atom transfer, or oxygen insertion reactions might be difficult or impossible to be imitated merely using direct electrochemical oxidations [8]. Among the important in vivo oxidative drug metabolism that are reportedly not mimicked with direct electrochemical oxidation are $\mathrm{N}$-oxidation, $\mathrm{O}$-dealkylation, and certain hydroxylation processes [8]. This issue highlights the immense need for development of electrochemical techniques that are versatile in promoting different oxidative metabolism pathways, and to have a practical tool for the fast and facile assessment of oxidative drug metabolism at early stages of new drug development.

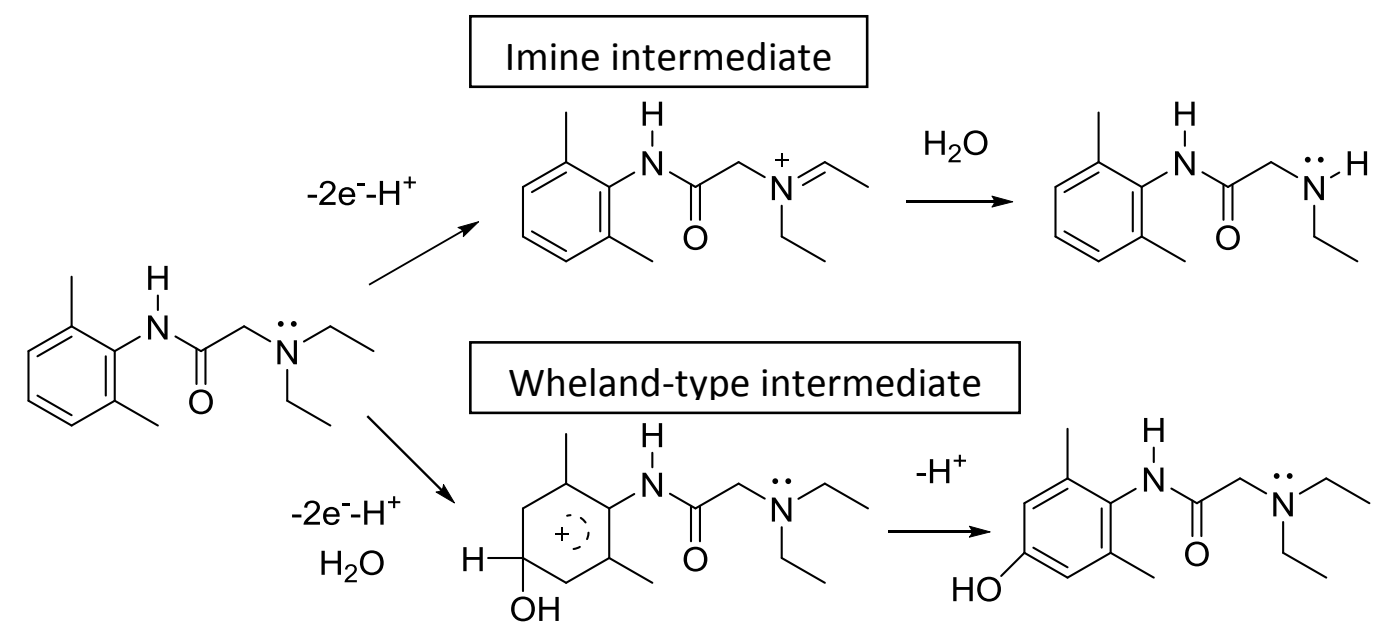

Scheme 1. Direct electrochemical oxidation of lidocaine (a test drug compound) resulting in the N-dealkylation of tertiary amine moiety, and para-hydroxylation reaction of the aromatic ring. $\mathrm{N}$-dealkylation proceeds through generation of an imine intermediate and hydrolysis, while para-hydroxylation proceeds through the generation of a Wheland-type intermediate and deprotonation.

Electrochemistry has shown already great promises in the mimicry of oxidative drug metabolism for a large number of drug models [9]. The reactions which are already imitated using electrochemistry are those reactions that are initiated by electron transfer. Direct electrochemical oxidation of lidocaine, as a test drug compound, results in two major oxidative metabolites, $N$-dealkylation, and para-hydroxylation [10]. The detailed mechanisms for these reactions are shown in Scheme 1. $\mathrm{N}$-dealkylation of tertiary amine 
compounds have already been studied extensively [11,12]. $N$-dealkylation reaction proceeds through electron transfer from nitrogen, formation an imine intermediate, hydrolysis, intra-molecular rearrangement, and $\mathrm{N}$-dealkylation [13]. Para-hydroxylation pathway follows an ECE mechanism with the first electron transfer, deprotonation, and second electron transfer that precede nucleophilic attack, and generating a Wheland-type intermediate in which substituents determines the final regioselectivity of the nucleophilic attack [14].

Electrochemical reactions are innately selective toward electron rich moieties, and a type of selectivity can be obtained by having controlled potential on the electrode surface, and a selectivity of reaction that can be obtained due to the onset potential of different reactions [7]. The electrochemical reactions can be integrated in microchips for a better application in industry [15]. Moreover, the electrochemical reactions can be utilized to perform preparative synthesis of highly interesting metabolites. This critical review presents the advantages of using hyphenated electrochemistry and mass spectrometry in the assessment of oxidative drug metabolism and discusses different tools such as the use of electrochemical generated reactive oxygen species, and the electrode surfaces modified with prophyrins or enzymes as candidates to be used for this purpose.

\section{Electrochemistry combined with mass spectrometry}

Although direct electrochemical oxidation confines the extension of using electrochemistry in the imitation of oxidative drug metabolism only to those reactions initiated by electron transfer reaction, it provides undisputable advantages to obtain insights in the mechanism of reactions. Direct coupling of a flow-through electrochemical cell with a mass spectrometry ushers in the near-real-time analysis of the oxidation products generated on the electrode surface. This facilitates the impact study of different electrochemical parameters such as applied potential, electrode material and solvent type on the distribution of reaction products. Reaction intermediates, and side products can be revealed and studied using hyphenated electrochemistry and mass spectrometry [12]. The addition of scavengers such as radical or cation scavengers would produce the evidence for the presence of certain short-living critical intermediates and their impact on the final product distribution [16]. The same approach can be valued for studying the reactivity of those intermediates with cell components [17], and their possible conjugated products with glutathione in phase II drug metabolism [18]. In the case of electrochemical oxidation of acetaminophen, and its counterpart in vivo oxidation, a toxic intermediates, $N$-acetyl-p-benzoquinone imine (NAPQI), can be generated that should be detoxified by conjugation with glutathione [18]. Interestingly, the detoxification of NAPQI with glutathione can follow two different nucleophilic pathways illustrated in Scheme 2. The conjugated products have different retention times and can be resolved with reverse phase chromatography, Figure 1A. The fragmentation patterns of the conjugated products are compared with the fragmentation of glutathione in Figure 1B. The fragmentation of the conjugated products is distinctive in nature possibly due to the steric hindrances imposed during fragmentations, Figure 1C, and Figure 1D. This in overall shows the advantage of using hyphenated electrochemistry and mass spectrometry in the exploration of different types of conjugates that might be difficult to be studied during in vivo experiments. 


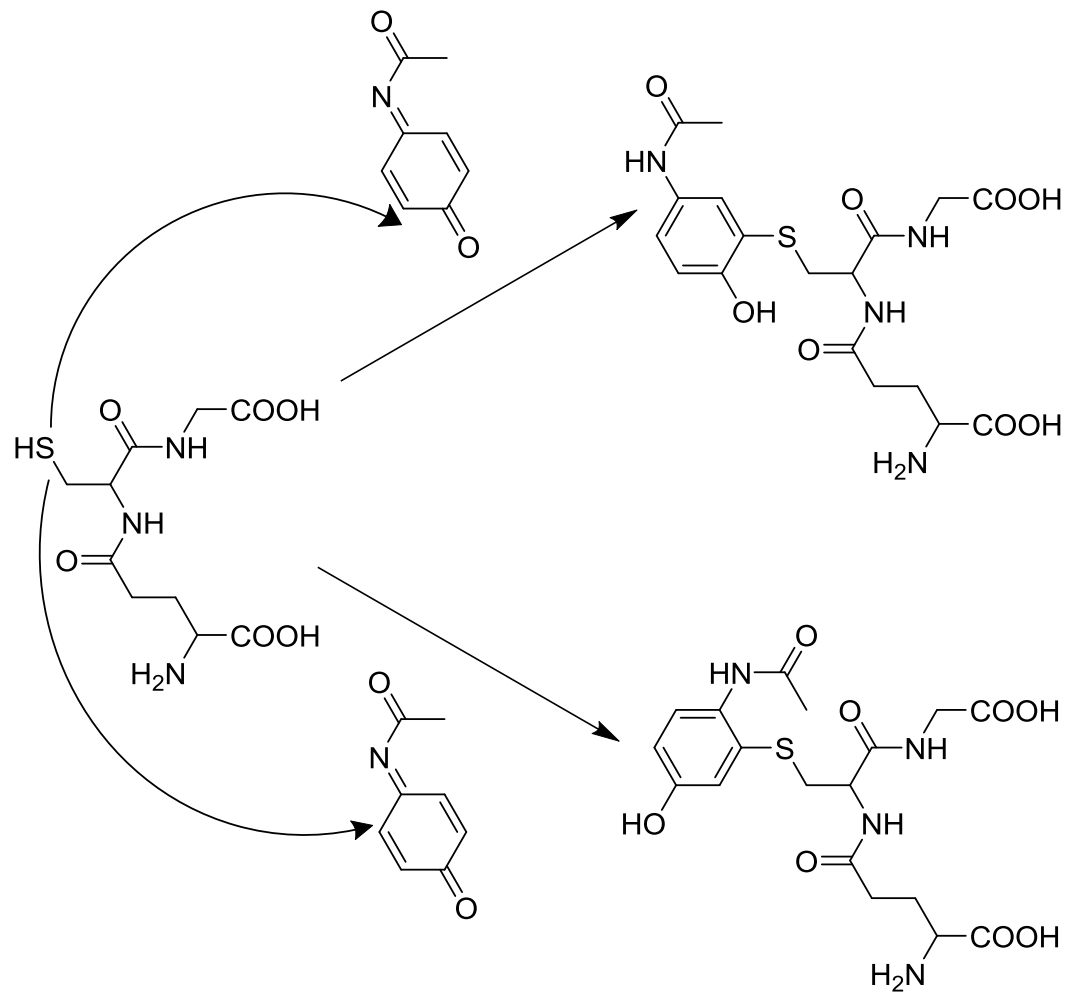

Scheme 2. Two different nucleophile reaction pathways for the glutathione and NAPQI which potentially result in the generation of two distinct conjugated products.

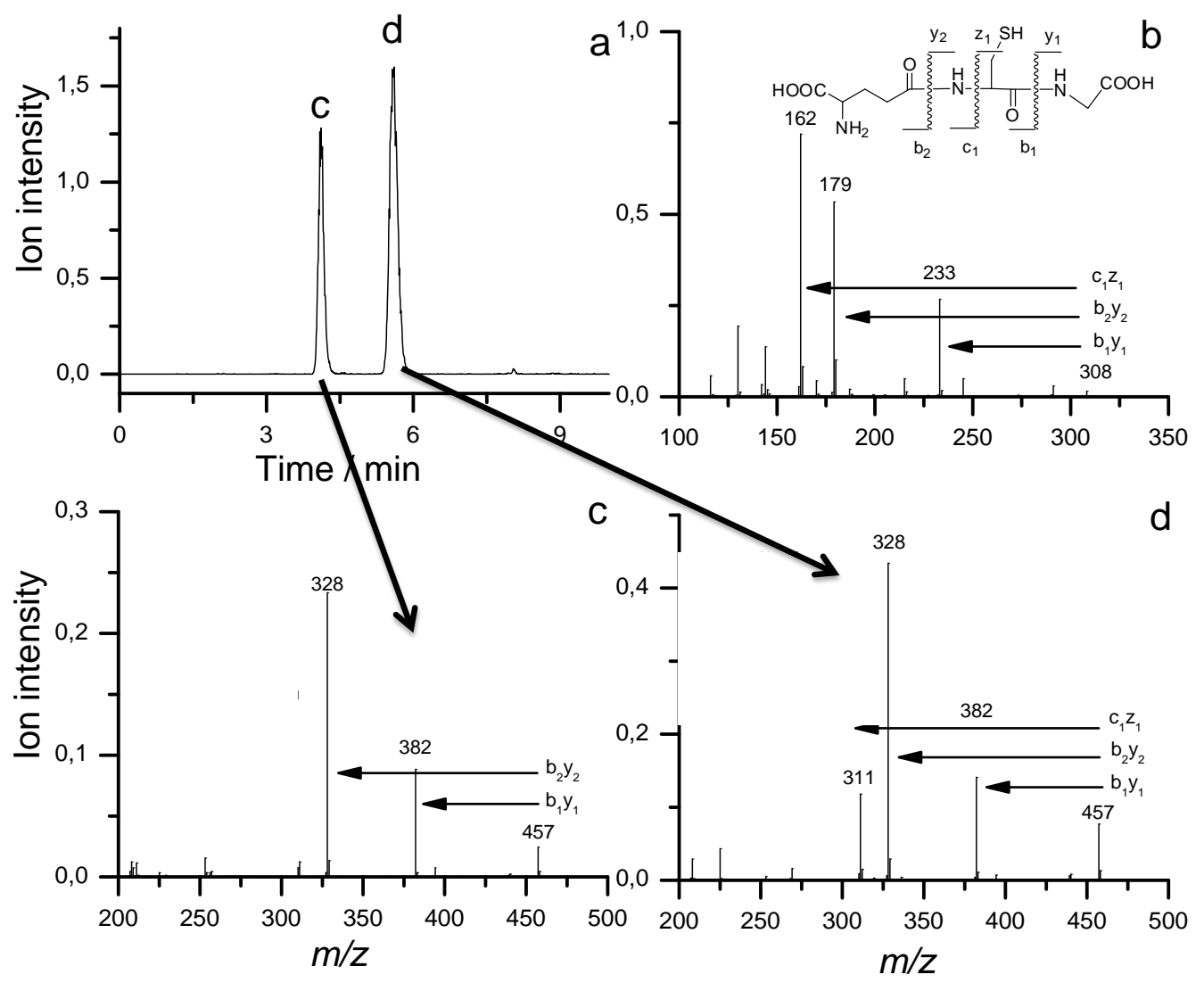

Figure 1. Conjugated products of glutathione and NAPQI that are resolved on a reverse phase chromatography column (a), MS/MS fragmentation pattern of glutathione with the fragments resulted from the break downs on two "by" positions and one "cz" position (b), corresponding fragmentation patterns for the first and second NAPQI and glutathione conjugate products separated on the reverse chromatography column (c and d). 


\section{Extension of electrochemistry to the reactions beyond electron transfer}

\subsection{Electrochemical pulses}

Electrochemical pulses consisting of repetitive oxidative and reductive steps have shown great promises in high yield and selective generation of drug metabolites [10]. For metallic electrodes such as gold and platinum, presence of an immediate reduction step would foster the reduction of oxide layer generated on the surface and removing the blocking reagents from electrode surface which in overall can result in higher oxidation yields. For the oxidation of lidocaine, hydroxylation pathway was enhanced by 50 times using electrochemical pulses with $1 \mathrm{~Hz}$ frequency [10]. In addition, using electrochemical potential pulses introduces a new parameter that can be used to tune the selectivity of electrochemical reactions. Interestingly, for lidocaine as a test drug compound, oxidations under higher frequencies favored $\mathrm{N}$ dealkylation pathway while for the longer pulse cycles this selectivity diverted to aromatic hydroxylation and $\mathrm{N}$-oxidation products [10].

Using electrochemical pulses not only can provide higher yields and selectivities, but also can promote reactions that were deemed impossible by constant potential oxidation. Biotransformation of phenacetin to acetaminophen was achieved using this approach [16]. The reaction included the generation of a cation intermediate, hydrolysis and reduction of NAPQI on the electrode surface to generate acetaminophen. Mechanistic studies revealed the generation of NAPQI in the presence of a strong nucleophile, and $p$ quinone as the final product of direct oxidation that was visualized using mass spectrometry by adding glutathione. There is much to perform to use the technique for a wide range of drug compounds and under different conditions that would be the matter of time and effort to be invested.

\subsection{Electrochemically generated reactive oxygen species}

Electrochemistry can be used to generate different reactive oxygen species on the surface. This type of reactions can be classified as indirect electrochemical reactions, in which electrochemistry generates reactive oxygen species to react with drug compounds. From this type of reactions, I will introduce the electrochemical formation of highly reactive hydroxyl radicals using electrochemically assisted Fenton reaction [19], generation of hydrogen peroxide [20], and oxidation of water on boron-doped diamond electrode (BDD) [21]. Hydroxyl radicals are highly reactive and easily promote the hydrogen atom transfer reactions; however, due to high reactivity, these species are not selective. A selective oxidation might be performed by electrocatalytic activation of hydrogen peroxide on platinum electrode surface to generate hypothetical platinum-oxo species that are capable of selective oxidation reactions. Scheme $\mathbf{3}$ shows the type of reaction products that each of the reactive species presented in this section would produce when reacted with lidocaine as a test drug compound.

Electrochemically assisted Fenton reaction: An earlier study indicated that the electrochemical reduction of $\mathrm{Fe}^{3+}$ to $\mathrm{Fe}^{2+}$ can promote the activation of hydrogen peroxide and the generation of hydroxyl radicals [19]. These radicals are highly reactive and less selective that lead to the generation of a wide range of product. A set of drug compounds were studied and it was explored that this type of reactions can imitate a wide range of oxidative drug metabolism [19]. In particular, reaction with hydroxyl radicals is capable of generating a wide range of hydroxylation products. A similar study for the oxidation of lidocaine also highlighted the fact that this type of reaction is capable of producing all types of in vivo metabolites of lidocaine (Scheme 3). Yet, due to the high reactivity of hydroxyl radicals, and their non-selective behavior, generation of a large quantity of unwanted side products is unavoidable. Though reaction with hydroxyl 
radicals could generate all known metabolites of lidocaine, there is clear presence of reaction products with the radical recombination with solvent molecules.

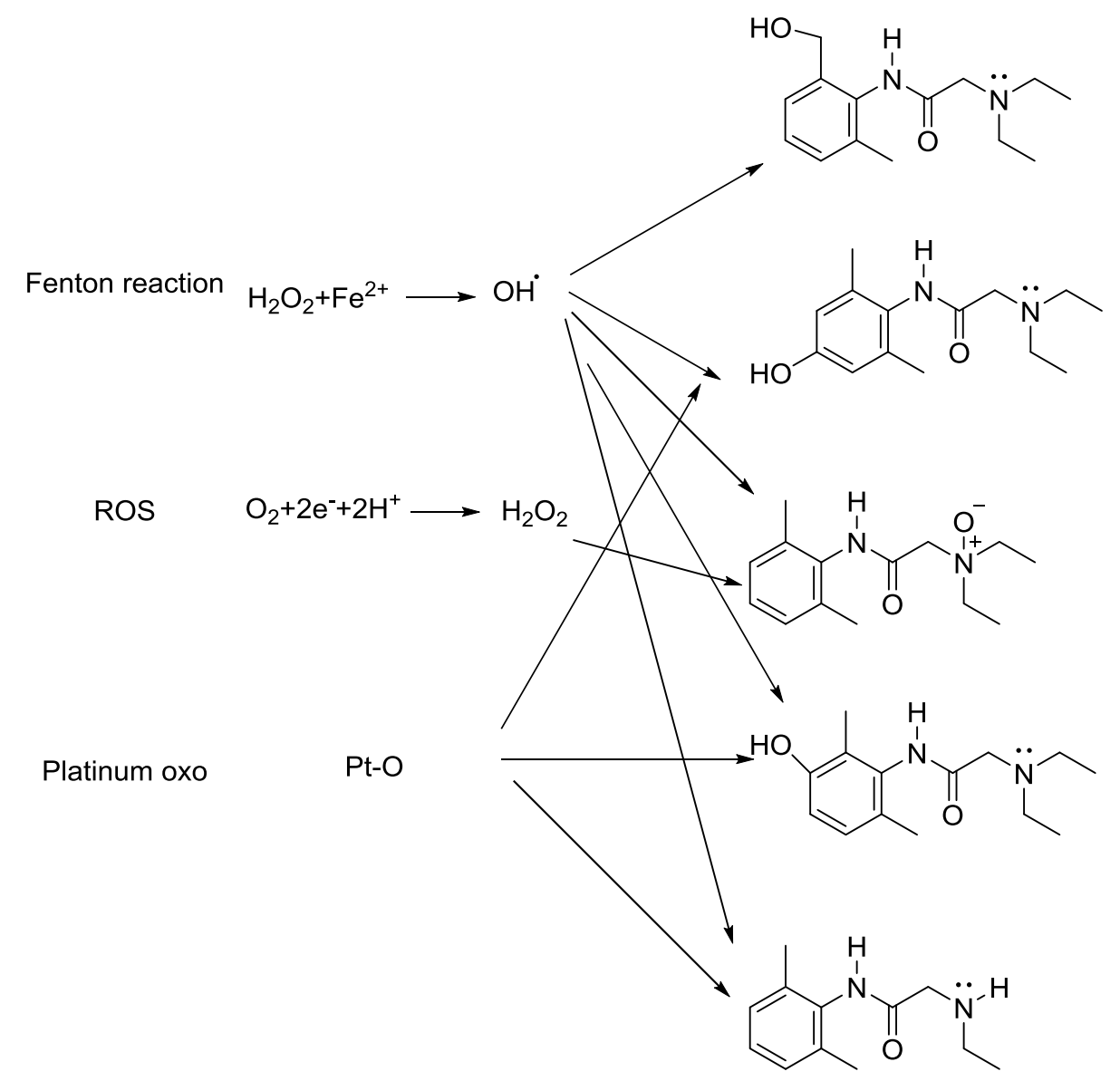

Scheme 3. The reaction of different types of electrochemically generated reactive oxygen species with lidocaine as a test drug compound and the corresponding metabolites generated from each of the reactions

Electrochemical reduction of molecular oxygen: electrochemical reduction of molecular oxygen from aprotic solvents can result in superoxide anions, that in the presence of residual water a chain of reactions can follow up to hydrogen peroxide [22]. Interestingly, superoxide anion can activate hydrogen peroxide in Haber-Weiss reaction type to produce highly reactive hydroxyl radicals. A past study to reduce molecular oxygen from acetonitrile in the presence of lidocaine generated a large quantity of $\mathrm{N}$-oxidation metabolite, while hydroxylation products were not detectable [13]. This highlights the fact that the main reactive species generated from the reduction of molecular oxygen under mentioned condition was hydrogen peroxide. Hydrogen peroxide reacts with the tertiary amine moiety of lidocaine and due to dehydrolysis $\mathrm{N}$ oxidation metabolite, a highly reactive metabolite, can be formed.

Electrocatalytic activation of hydrogen peroxide: Electrocatalytic behavior of platinum electrodes in the oxidation of hydrogen peroxide is a well-known phenomenon. Yet, the generation of hypothetically reactive intermediates during electrocatalytic activation of hydrogen peroxide on platinum electrode to promote selective oxidation pathways is yet to be explored. In a recent study, we showed that oxidation of hydrogen peroxide on a platinum electrode can produce both meta and para isoforms of aromatic hydroxylation of lidocaine, in the absence of benzylic hydroxylation product, Scheme 3 [20]. This helped us to suggest a more selective oxidation reaction in the absence of hydroxyl radicals due to generation of hypothetical platinum-oxo species that are capable of promoting selective oxygen insertion reactions. $\mathrm{A}$ 
thorough mechanistic study needs to be performed, and the results of this relatively simple approach can widen the selective oxidation pathways of drug candidates.

BDD electrodes: oxidation of water on BDD electrode can generate hydroxyl radicals capable of nonselective oxidation reactions. BDD electrode due to high polarization of molecular oxygen evolution has a wide range of applied potential window for the oxidation of water that results in the formation of hydroxyl radicals. This approach due to its simplicity has gained an ever increasing attention in the mimicry of oxidative drug metabolism, as well as electrochemical cleavage of peptides and proteins [21].

\subsection{Electrochemical mimicry with porphyrins}

The prosthetic group of Cytochrome P450s is an iron protoporphyrin, and hence metalloporphyrins can be in general good surrogates to mimic the catalytic behavior of Cytochrome P450s [23]. On a mechanistic view, metallo-oxo species can be formed either with reaction with a mono-oxygen atom donor such as $m$ CPBA, as shown in Scheme 4, or with hydrogen peroxide after dehydrolysis that are considered shunt pathways [3]. By using electrochemistry, it is possible to activate molecular oxygen in a two electron, two proton transfer reaction steps, as shown with the main catalytic cycle in Scheme 4 [24]. The consecutive electron and proton transfers result in a heterolytic activation of oxygen-oxygen bond and generation of metallo-oxo species. The oxygen transfer reaction would re-generate the initial state of the porphyrin. In the case of electrochemistry, there is a need for a relatively strong acid such as acetic acid to perform the protonation, in addition to an axial coordinator that is usually 1-methyl imidazole [25].

The mechanism was verified by the cyclic voltammetry of hemin from DMSO, $0.1 \mathrm{M}$ TBAP, under argon atmosphere, that shows a single reversible electron transfer, corresponding to the first electron transfer, shown in Figure 2 [26]. Interestingly, in the presence of dissolved molecular oxygen, this single redox peak changes to two distinct electron transfer peaks, supporting well the mechanism shown in Scheme 4. The second cycle under oxygen atmosphere shows the absence of first electron transfer, this indicates that the species generated during the first cycle is stable and there is not $\mathrm{Fe}^{2+}$ available for re-reduction during the following cycle. In the presence of a strong acid, $100 \mathrm{mM}$ acetic acid, instead of two separate peaks there is a single broad peak that is totally irreversible, and reproduces in the consecutive scans. This may indicate the generation of reactive species and their release. 


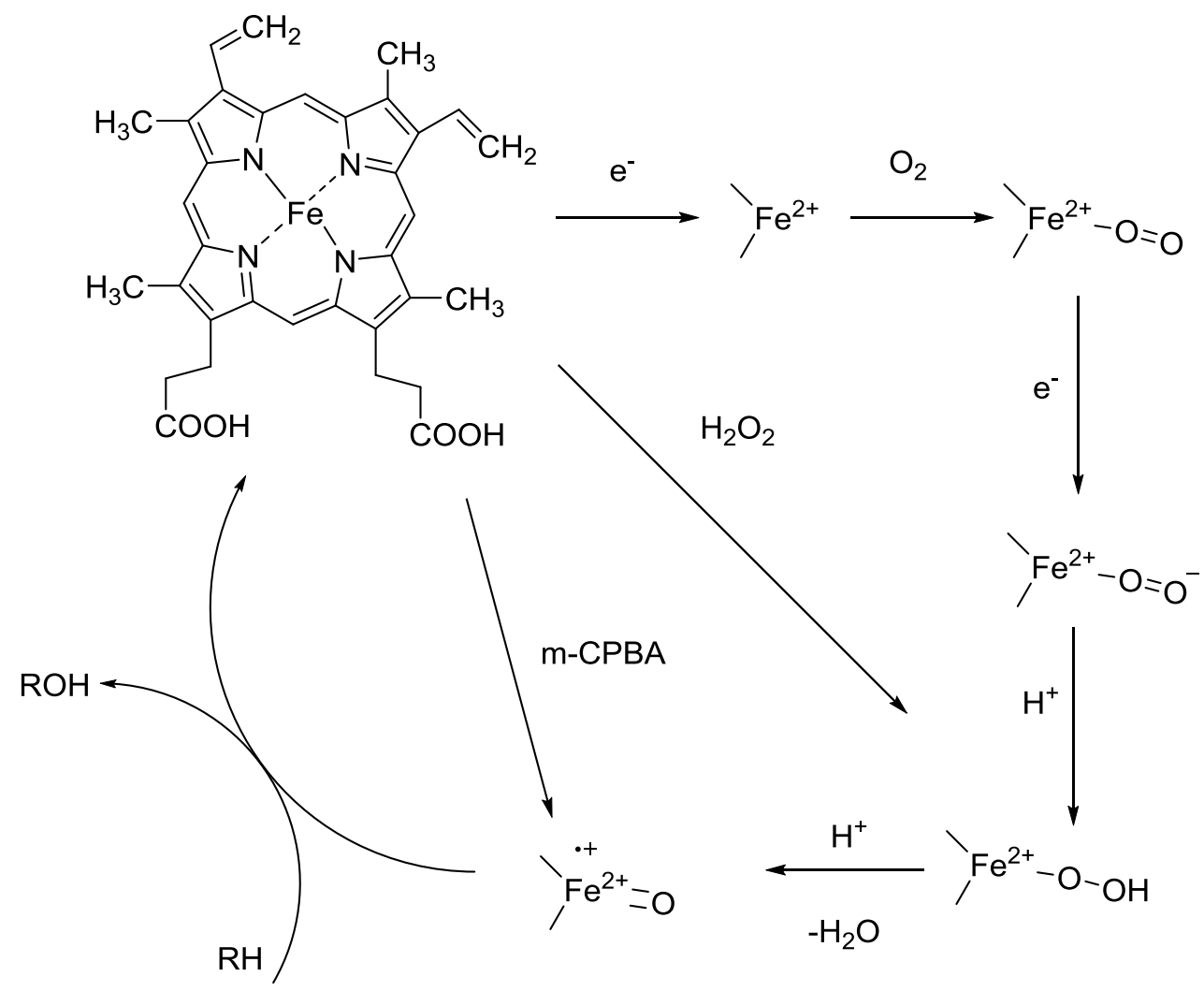

Scheme 4. Catalytic activation of molecular oxygen with hemin, as a mimetic surrogate for Cytochrome P450s, that includes two electron transfer, and two proton transfer steps, resulting in the formation of highly reactive oxo ferryl radical cations. In addition, there are two shunt pathways by either reaction with hydrogen peroxide, or a mono oxygen atom donor such as $m-C P B A$.

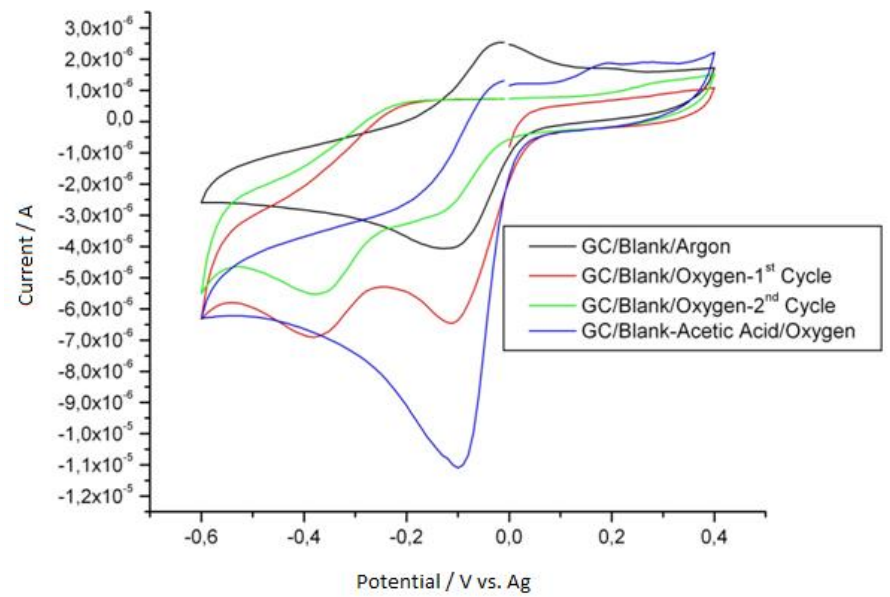

Figure 2. Cyclic voltammograms of hemin on the glassy carbon electrode from DMSO, $0.1 \mathrm{M} T B A P$, under argon atmosphere (black), the first cycle under oxygen atmosphere and second cycle (red and green respectively), and the cycle under oxygen atmosphere and in the presence of $100 \mathrm{mM}$ acetic acid (blue line) [26].

The product distribution depends on the type of metallic center and presence of electron-withdrawing substituent on the porphyrin ring. Generally, having stronger electron-withdrawing substituents on the porphyrin ring makes the reacting metallo-oxo species more reactive and promotes more difficult reactions. A comparative study revealed that while manganese porphyrin in the presence of $m$-CPBA only generates $\mathrm{N}$-dealkyaltion product, under the same reaction condition, fluorinated manganese porphyrin 
generates all the hydroxylation products, with a much larger quantity of $\mathrm{N}$-dealkylation product. Using asymmetric porphyrins can also result in stereospecific promotion of oxidative drug metabolism [27].

The metalloporphyrins can be immobilized on the electrode surface through electropolymerization $[28,29]$, or through self-assembled monolayers [9]. Figure $3 \mathbf{A}$ shows the hemin accumulated on the electrode surface, with a similar electrochemical behavior as hemin from solution. The significant difference is that there is a higher current density in the presence of a strong proton donor that might be due to the direct reduction of electrochemically generated reactive oxygen species.

The main issue with the immobilized porphyrin electrochemistry is the instability of electrode surface that might be deteriorated after few cycles. Figure 3B shows that the hemin from electrode surface is not stable and would dissolved to the solvent after few cycles of redox potentials.

On the other hand using single atomic layer of porphyrins on the electrode surface has proven advantages for spectroscpoic purposes [30], but unfortunately the stability of surface during oxidation reaction is a perplexing challenge to overcome. Given the wide variety of metallopoprphyrins with different metallic centers, substituents, and stereochemistry this can be a highly interesting topic of development for further mimicry studies of oxidative drug metabolism.
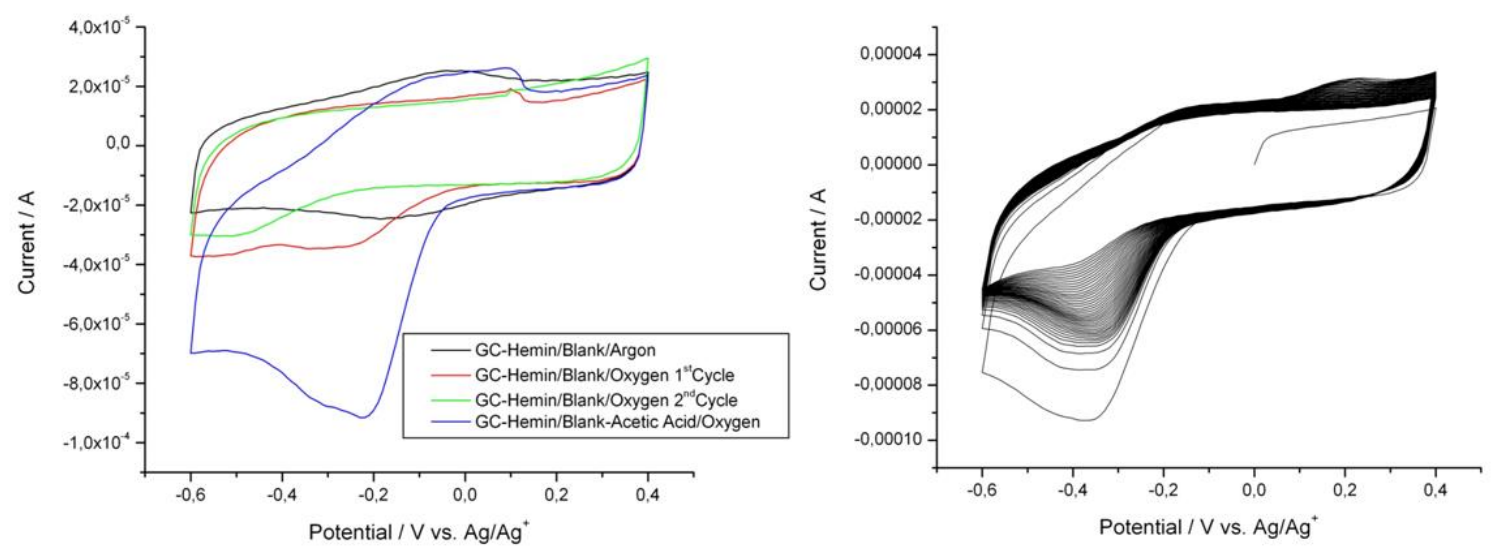

Figure 3. (A) Cyclic voltammograms of hemin accumulated on a glassy carbon electrode under argon atmosphere (black), the first cycle under oxygen atmosphere and second cycle (red and green respectively), and the cycle under oxygen and in the presence of acetic acid (blue line). (B) repetitive cycles of hemin accumulated on the glassy carbon surface in DMSO, 0.1 M TBAP, $100 \mathrm{mM}$ acetic acid [26].

\subsection{Electrochemistry combined with electrode surfaces modified with enzymes}

A highly stereospecific and chemoselective mimicry can be achieved using synthetic heme peptides and enzymes, such as peroxidases and Cytochrome P450s [9]. Using an enzyme as a mimetic model is challenging due to the complications with charge transfer reactions, protein stability and inactivation due to immobilization. Using isolated Cytochrome P450s requires additional reductase cofactor. A protein engineering strategy might also help to obtain sufficient interaction of the enzyme with electrode surfaces using an enzyme reductase fused protein [31]. This is due to the fact that the enzyme immobilization should not inactivate or change the enzyme behavior. The possibility of using electrodes modified with enzymes in micro fluidic can add value to their industrial applications [32]. Different strategies using enzymes in mimicry of oxidative drug metabolism are already discussed in an earlier review article [9]. 


\section{Conclusions}

Hyphenated electrochemistry and mass spectrometry can gain a greater momentum in the practical senses for the assessment of oxidative drug metabolism. However, there is a great need to extend the application of electrochemistry to the reactions initiated by those other than electron transfer. This brief review presented a few promising electrochemical approaches that can help to achieve this goal. Though electrochemically generated reactive species showed a great promise for the oxidation of lidocaine as a test drug compound, high reactivity of hydroxyl radicals and their non-selective behavior would refrain their use. Electrochemically generated platinum-oxo species, though showed specific oxygen insertion reactions, need to be studied in the context of different compounds. The metalloporphyrins are interesting due to promoting highly selective reaction pathways with a wide selection of metallic centers and substituents; however, there is a need to develop stable electrode modifications. Using enzymes as mimetic models can promote sterospecific and chemoselective reactions. Yet, there are complications with electron transfer to enzymes, degradation, and deactivation due to immobilization.

\section{References}

[1] H. Faber, M. Vogel, U. Karst, Analytica Chimica Acta, 834 (2014) 9-21.

[2] M. Vogel, U. Karst, Analytical and Bioanalytical Chemistry, 403(2) (2012) 333-334.

[3] P.R. Ortiz de Montellano, Cytochrome P450: Structure, Mechanism, and Biochemistry. Third ed 2005, New York: Kluwer Academic/Plenum Publishers.

[4] S. Shaik, D. Kumar, S.P. de Visser, A. Altun, W. Thiel, Chemical Reviews, 105(6) (2005) 2279-2328.

[5] S. Shaik, S. Cohen, Y. Wang, H. Chen, D. Kumar, W. Thiel, Chemical Reviews, 110(2) (2010) 949-1017.

[6] B. Meunier, S.P. de Visser, S. Shaik, Chemical Reviews, 104(9) (2004) 3947-3980.

[7] U. Jurva, H.V. Wikström, A.P. Bruins, Rapid Commun. Mass Spectrom., 14(6) (2000) 529-533.

[8] U. Jurva, H.V. Wikström, A.P. Bruins, Rapid Commun. Mass Spectrom., 17(8) (2003) 800-810.

[9] E. Nouri-Nigjeh, R. Bischoff, A.P. Bruins, H.P. Permentier, Current Drug Metabolism, 12(4) (2011) 359371.

[10] E. Nouri-Nigjeh, H.P. Permentier, R. Bischoff, A.P. Bruins, Analytical Chemistry, 83(14) (2011) 55195525.

[11] L.R. Hall, R.T. Iwamoto, R.P. Hanzlik, The Journal of Organic Chemistry, 54(10) (1989) 2446-2451.

[12] U. Jurva, P. Bissel, E.M. Isin, K. Igarashi, S. Kuttab, N. Jr Castagnoli, Journal of American Chemical Society, 127(35) (2005) 12368-12377.

[13] E. Nouri-Nigjeh, H.P. Permentier, R. Bischoff, A.P. Bruins, Analytical Chemistry, 82 (2010) 7625-7633.

[14] L. Eberson, K. Nyberg, Accounts of Chemical Research, 6(3) (1973) 106-112.

[15] M. Odijk, A. Baumann, W. Lohmann, F.T.G. van den Brink, W. Olthius, U. Karst, Lab on a Chip, 9(12) (2009) 1687-1693.

[16] E. Nouri-Nigjeh, R. Bischoff, A.P. Bruins, H.P. Permentier, Analyst, 136(23) (2011) 5064-5067.

[17] W. Lohmann, U. Karst, Analytical Chemistry, 79(17) (2007) 6831-6839.

[18] W. Lohmann, U. Karst, Analytial and Bioanalytical Chemestry, 386(6) (2006) 1701-1708.

[19] U. Jurva, H.V. Wikström, A.P. Bruins, Rapid Communications in Mass Spectrometry, 16(20) (2002) 1934-1940.

[20] E. Nouri-Nigjeh, A.P. Bruins, R. Bischoff, H.P. Permentier, Analyst, 137(20) (2012) 4698-4702.

[21] J. Roeser, N.F.A. Alting, H.P. Permentier, , A.P. Bruins, R. Bischoff, Analytical Chemistry, 85(14) (2013) 6626-6632.

[22] J. Wilshire, D.T. Sawyer, Accounts of Chemical Research, 12(3) (1979) 105-110. 
[23] T. Johansson, L. Weidolf, U. Jurva, Rapid Commun Mass Spectrom, 21(14) (2007) 2323-2331.

[24] S.R. Bell, J.T. Groves, Journal of the American Chemical Society, 131(28) (2009) 9640-9641.

[25] S.E. Creager, S.A. Raybuck, R.W. Murray, Journal of the American Chemical Society, 108(14) (1986) 4225-4227.

[26] E. Nouri-Nigjeh, H.P. Permentier, R. Bischoff, A.P. Bruins, 59th Annual Meeting of the International Society of Electrochemistry, Seville, Spain (2008).

[27] K. Chen, L. Que Jr, Chemical Communications, (1999) (15): p. 1375-1376.

[28] F. Bedioui, J. Devynck, C. Bied-Charreton, Accounts of Chemical Research, 28(1) (1995) 30-36.

[29] F. Bedioui, J. Devynck, C. Bied-Charreton, Journal of Molecular Catalysis A: Chemical, 113(1-2) (1996) 3-11.

[30] A. Bonifacio, D. Millo, P. Keizers, R. Boegschoten, J. Commandeur, N. Vermeulen, C. Gooijer, G. van der Zwan, Journal of Biological Inorganic Chemistry, 13(1) (2008) 85-96.

[31] S.J. Sadeghi, R. Meirinhos, G. Catucci, V.R. Dodhia, G.D. Nardo, G. Gilardi, Journal of the American Chemical Society, 132(2) (2009) 458-459.

[32] A. Fantuzzi, E. Capria, L.H. Mak, V.R. Dodhia, S.J. Sadeghi, S. Collins, G. Somers, E. Hug, G. Gilardi, Analytical Chemistry, 82(24) (2010) 10222-10227.

(C)2014 by the authors; licensee IAPC, Zagreb, Croatia. This article is an open-access article distributed under the terms and conditions of the Creative Commons Attribution license (http://creativecommons.org/licenses/by/3.0/) (cc)) EY 